\title{
QFT Control for Vibration Reduction in Structures Equipped with MR Dampers
}

\author{
Mauricio Zapateiro, Ningsu Luo, Hamid Reza Karimi
}

\begin{abstract}
This short paper addresses the problem of designing a QFT (Quantitative Feedback Theory) based controllers for the vibration reduction in a 6-story building structure equipped with shear-mode magnetorheological dampers. A new methodology is proposed for characterizing the nonlinear hysteretic behavior of the MR damper through the uncertainty template in the Nichols chart. The design procedure for QFT control design is briefly presented.
\end{abstract}

\section{INTRODUCTION}

Most of active and semiactive structural control strategies are based on the idea of attenuating vibrations or maintaining them within certain acceptable range, when external forces such as earthquakes or strong winds act on the structures [1]. The controller design is done usually by considering that the structural model and its associated parameters are known or uncertain but bounded. The effectiveness of control system depends on the precision of estimated or measured variables (displacement, velocities, etc). Due to the fact that the behavior of controlled structures depends not only on the magnitude of the external excitation but also on its frequency modes, the modal frequency control is of a big interest for achieving the safety and comfort of the structures. However, the controller design becomes very difficult when parametric uncertainty and nonlinear dynamics are involved in a wide range of frequencies.

This short paper deals with the problem of vibration control of a class of building structures equipped with MR dampers. A new design methodology is presented by applying Quantitative Feedback Theory based robust control to the vibration reductions at the critical resonant frequencies, in which the hysteretic nonlinearity is represented by some uncertainty template within the Nichols chart. Finally, some concluding remarks are made.

\section{PROBLEM FORMULATION}

The theoretical research is carried out on a prototype of building structure of 6 stories, single bay, steel frame and $188 \mathrm{~cm}$ tall and has a mass of $147 \mathrm{~kg}$, distributed uniformly among the floors. A couple of MR dampers are installed between the ground and first floor, and the other couple

Dr. Ningsu Luo is with Institute of Informatics and Applications, University of Girona, Spain (corresponding author: phone: +34 972 418888, Fax: +34 972 418259). ningsu. luo@udg. edu

Mr. Mauricio Zapateiro is with Institute of Informatics and Applications, University of Girona, Spain. mauricio.zapateiro@udg.edu

Dr. Hamid Reza Karimi is with Institute of Informatics and Applications, University of Girona, Spain. hrkarimi@eia.udg.edu between the first and second floors of the structure. Control forces applied to the structure are sensed by means of force transducers placed in series with the MR damper.

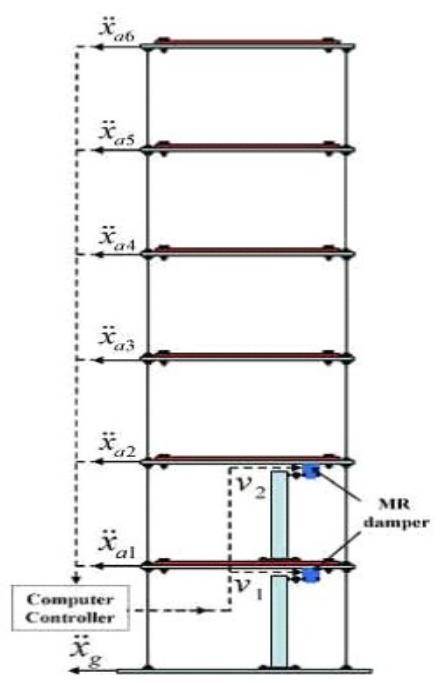

Fig. 1. Semiactivelly controlled structure

The MR damper used in the experiments is a prototype device fabricated by the Lord Corporation. It consists of two steel parallel plates with the dimension of $4.45 \mathrm{~cm} \times$ $1.9 \mathrm{~cm} \times 2.5 \mathrm{~cm}$. Damper force is generated when the moving plate, coated with a thin foam saturated with MR fluid, slides between the two parallel plates. An electromagnet, consisting of a coil installed at one end of the devices, produces the magnetic field applied on the MR fluid of the saturated foam. A maximum force of $29 \mathrm{~N}$ can be generated by each MR damper.

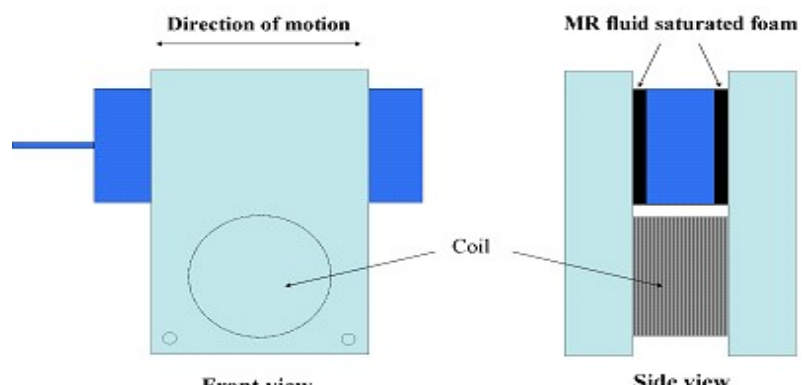

Fig. 2. MR damper configuration 


\section{QFT CONTROLLER DESIGN}

QFT based robust control is one of well known effective frequencial techniques for controlling different types of practical processes [2]-[3]. As the most important properties of QFT, the procedure of controller design is transparent and systematic, and it is relatively easy to include uncertain factors in the performance's specifications. Thus, the quantitative formulation of plant uncertainty and different performance specifications are essential for the feedback control.

The basic developments with QFT theory are focused on the control design problem for uncertain Linear Time Invariant (LTI) systems. Consider the system of the figure 3 as the basic structure, in which $\boldsymbol{R}$ represents the command input set, $\boldsymbol{P}$ the plant set and $\boldsymbol{T}$ the object transfer function set. For each $R$ in $\boldsymbol{R}, P$ in $\boldsymbol{P}$, the closed-loop output will be $Y(s)=T(s) R(s)$ for some $T$ in $\boldsymbol{T}$. For a large class of such LTI problems, QFT based design is executable; i.e., a pair of controllers $F(s)$ and $G(s)$ can be found to guarantee that $Y(s)=T(s) R(s)$. Suppose that the plant $P$ is an uncertain but known member of the set $\boldsymbol{P}$. The designer is free to choose the prefilter $F$ and the loop compensator $G$ in order to ensure that the system transfer function $T=$ $F P G /(1+H P G)$ satisfy the assigned specifications.

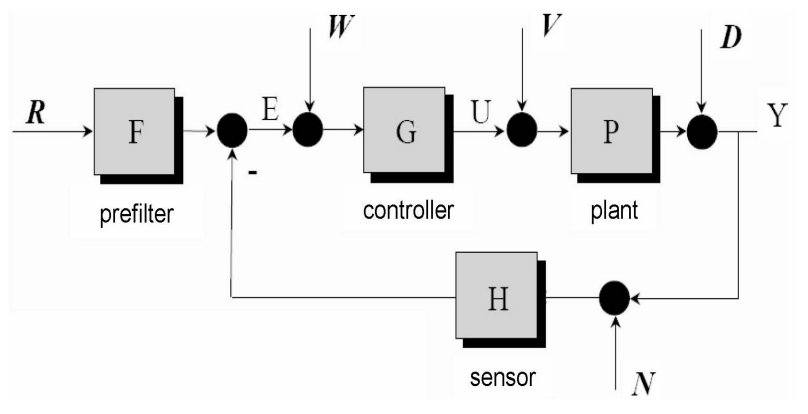

Fig. 3. Basic structure of control system

Structural control methodologies based on the Quantitative Feedback Theory (QFT) for vibration reduction in linear structures were developed during the recent years [4]-[5]. This work considers a structure semiactivelly controlled by MR dampers as described in the section II. The dynamics of the building structure is linear, which can be represented by the following equations:

$$
\boldsymbol{M}_{s} \ddot{\boldsymbol{x}}+\boldsymbol{C}_{s} \dot{\boldsymbol{x}}+\boldsymbol{K}_{s} \boldsymbol{x}=\boldsymbol{\Lambda} \boldsymbol{f}-\boldsymbol{M}_{s} \boldsymbol{\Gamma} \ddot{\boldsymbol{x}}_{g}
$$

with $\boldsymbol{x}$ being a vector of relative displacement of the structural floors, $\ddot{\boldsymbol{x}}_{g}$ the ground acceleration, $\boldsymbol{\Gamma}$ a column vector of ones, and $\boldsymbol{\Lambda}$ a vector determined by the placement of the MR dampers in the structure, and $\boldsymbol{M}_{s}, \boldsymbol{C}_{s}$ and $\boldsymbol{K}_{s}$ the mass, damping and stiffness matrices. $\boldsymbol{f}=\left[f_{1}, f_{2}\right]^{T}$ is a vector of measured control forces generated by the MR dampers,

$$
f_{i}=\delta \dot{x}_{i}+\alpha z_{i}
$$

The nonlinear hysteretic behaviour of the shear-mode MR dampers $(\mathrm{i}=1,2)$ used in this research can be described by the following Bouc-Wen model [6]:

$$
\dot{z}_{i}=-\gamma\left|\dot{x}_{i}\right|-\beta \dot{x}_{i}|z|+A \dot{x}_{i}
$$

where $z$ is an evolutionary variable that accounts for the dependence of the historical responses, $\gamma=300, \beta=300$ and $A=120$.

In general, the QFT based control design requires that the dynamics of the plant $P$ be LTI. Since the semiactive control system dealt with in this paper contains the nonlinear MR dampers, it is not possible to make the LTI control design directly. In order to solve this problem caused by the hysteretic nonlinearity of the MR dampers, it is proposed in this research work to represent the hysteretic cycle as a LTI nominal system $P_{H N}$ bounded by a set of linear systems $P_{H i}$ $(\mathrm{i}=1,2,3,4)$, which can be treated as the model uncertainty regarding to the LTI nominal system $P_{H N}$.

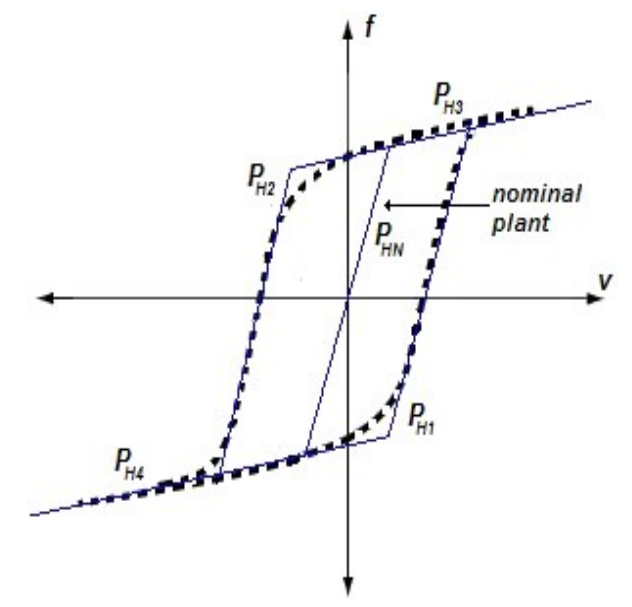

Fig. 4. LTI bounded characterization of hysteresis cycle

The Logarithmic Complex Plane (Nichols Chart) is a highly transparent medium for visualizing the design procedure for the above purpose. It consists of loci of constant $M=|T|$, and $\operatorname{Arg}(T)$ in the logarithmic $L$ plane: Angle $(L)$ in degrees, Magnitude $(L)$ in db $(20 \log 10|L|)$. Suppose $H=1$, the QFT based control design will be done step by step to obtain the prefilter $F$ and the compensator $G$.

In the first step, the characterization of the plant uncertainty is done by templates $(P)$ and loop templates $(L=P G)$. In this step, the uncertain nonlinearity existed in the plant $P(s)$ is displayed on the Nichols Chart. At each frequency, $P(j \omega)$ will be a complex number. Because of plant uncertainty, the plant template $P(j \omega)$ is a set of complex numbers corresponding to a set of uncertain plants at each frequency $\omega$. Since $L=P G$ varies with $P$, a nominal $P_{N}$ is chosen to give a nominal $L_{N}=P_{N} G$. Note that the template of $L=P G$ is isometric to the template of $P$. The template $L(j \omega)$ is obtained by shifting the plant template $P(j \omega)$, by Angle $(G(w))$ horizontally, by $|G(j \omega)|$ vertically. Its shape and size are the same as the template $(P)$.

In the second step, the bounds on the nominal $L_{N}(j \omega)$ are obtained. In this step, the design tool is the nominal 
loop template $L_{N}(j \omega)$. By making it the smallest possible, specifications can be satisfied and quantitative control is obtained. Simply manipulate the plant $=$ loop template on the Nichols chart, until (at any fixed angle), the minimum $\left|L_{N}\right|$ is found, which satisfies the specifications. It is seen that this $\left|L_{N}\right|_{\text {min }}$ is a function of $\operatorname{Angle}\left(L_{N}\right)$. The resulting curve is called the bound $B(j \omega)$ on $L_{N}(j \omega)$, as shown in the Nichols Chart of figure 5 . The procedure to find the bounds at all frequencies is made by programming it in the computer.

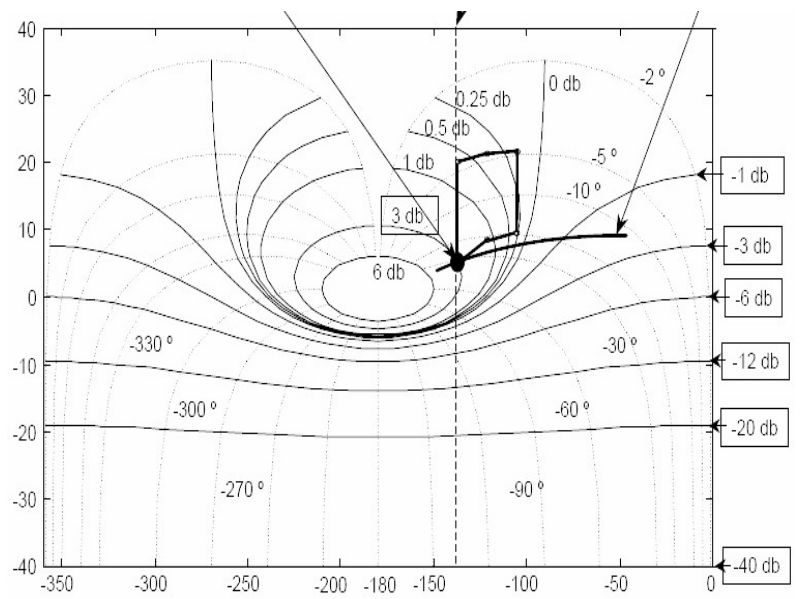

Fig. 5. Drawing the bound $B\left(j \omega_{i}\right)$

Afterwards, the Universal High-Frequency Bound $U H \omega B$ is found. For $\omega>>1$, the templates $P(j \omega)$ are almost vertical lines. The fact that in the higher frequency the plant template is a fixed vertical line, leads to universal high frequency bound $(U H \omega B)$. In this frequency range the allowed $M$ variation is larger than the plant uncertainty.

The next design step consists in finding $L_{N}(j \omega)$ which satisfies its bounds $B(j \omega)$. It is done by using the MATLAB coded QFT toolbox supplied by the Terasoft Inc. The idea is to design a controller $G(s)$, which allows the loop function $L(s)=G(s) P(s)$ to accomplish the specifications defined by means of the bounds. By considering the most restrictive conditions from this set of bounds, the resulting bound is found by intersecting the constrained bounds. By taking into account such bound, the optimal design is ensured if for each $\omega_{i}, L_{N}\left(j \omega_{i}\right)$ is in its corresponding bound. In a practical way, $L_{N}(j \omega)$ is designed to be as near as possible to $B(j \omega)$, in order to keep minimum the bound width of $T(j \omega)$. By using the QFT toolbox, one possible loop $L_{N}$ that accomplishes the specifications is shown in figure 6.

The final design step is to obtain the controller $G(s)$ and prefilter $F(s)$. The controller $G(s)$ is available from $L_{N}$ $\left(G(s)=L_{N}(s) / P_{N}(s)\right)$ to assure that the allowed variation in $|T(j \omega)|, M_{\max }-M_{\min }=\delta(\omega)$ be satisfied for all $\omega$. Since $|T|=|F| M$, the function of $F$ is to fit to the actual object set variation inside the $M$ specification. This is achieved by assigning bounds on $|F(j w)|: \log F_{\min }(j \omega)<$ $\log F(j \omega)<\log F_{\max }(j \omega)$ and additionally to ensure that

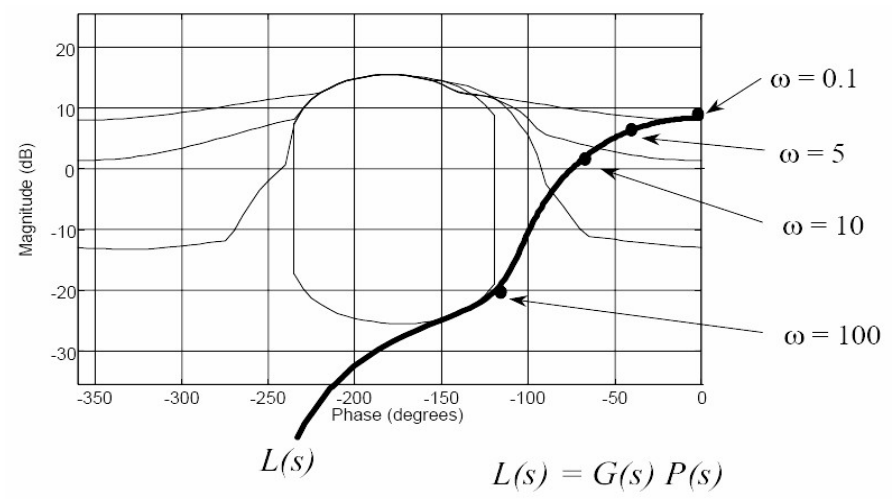

Fig. 6. Possible loop L

\section{CONCLUSION}

As a novelty in this paper, the QFT control design technique has been introduced in structural control for the vibration reduction in a building structure equipped with MR dampers. A simple characterization of the nonlinear hysteretic cycle is proposed to represent the whole plant in the Nichols chart that contains the uncertainty template, by using the bounded LTI systems based on the LTI nominal one. The advantage of using QFT in structural control is that the design is made in frequency, which is a very important issue, taking into account that disturbances are uncertain and vibrations can be produced in the structure if it is excited at the natural frequency. The QFT based control design has taken into account this consideration. The applicability and effectiveness of the technique is demonstrated by means of a numerical example (omitted here due to the space limitation).

\section{ACKNOWLEDGEMENT}

This work has been partially funded by the European Regional Development Fund and the Commission of Science and Technology of Spain (CICYT) through the research project DPI-2005-08668-C03 and by the Government of Catalonia through SGR00296. The first and third authors are grateful to the FI Grant of the Department for Innovation, University and Enterprise of the Government of Catalonia and to the grant of Juan de la Cierva program of the Ministry of Education and Science of Spain, respectively.

\section{REFERENCES}

[1] Spencer Jr., B.F. and M.K. Sain, Controlling buildings: a new frontier in feedback, IEEE Contr. Syst. Magazine: Special Issue on Emerging Technologies, 19-35, 1997.

[2] I. Horowitz. Fundamental Theory of Linear Feedback Control Systems. Transactions IRE on Automatic Control. AC-4. 1959 [3]

[3] A. Baños and I. Horowitz, Nonlinear quantitative stability. International Journal of Robust Nonlinear Control. v14. 289-306, 2004.

[4] N. Luo, R. Villamizar, J. Vehí, Quantitative Feedback Theory (QFT): application to structural control, China-Japan-US Symposium on Health Monitoring and Control of Structures, Dalian, China, 2004.

[5] R. Villamizar, N. Luo, J. Vehí, J. Rodellar, Active and semiactive QFT control for the structural vibration attenuation, Conference on Mechanical Vibration and Noise (MOVIC), St Louis, USA, 2004.

[6] F. Ikhouane, J. Rodellar, Systems with hysteresis: Analysis, identification and control using the Bouc-Wen model. John Wiley \& Sons, 2007. 